de in der Praxis nie erwartet werden. Im Gegensatz dazu waren in der SWOG-Studie alle Patientinnen Östrogenrezeptor positiv.

5. Weiter erschwert wird die Interpretation noch dadurch, dass je die Hälfte der Patientinnen prä- bzw. postmenopausal waren. Die Sequenz der Chemo- und der endokrinen Therapie kann aber durchaus in der Prämenopause zu anderen Ergebnissen führen als in der Postmenopause. Im Gegensatz dazu waren in der SWOGStudie alle Patientinnen postmenopausal. Vor diesem Hintergrund kann die vorliegende Arbeit nicht praxisändernd sein, wohl wissend, dass eine Überlegenheit der sequenziellen chemoendokrinen Therapie im wissenschaftlichen Sinne nicht bewiesen ist. Dies gilt umso mehr vor dem Hintergrund, dass in der Postmenopause heute ausnahmslos fast alle Östrogenrezeptorpositiven Patientinnen auch eine Therapie mit einem Aromatase-Inhibitor erhalten. Zum „Timing" der chemoendokrinen Therapie liegen für Aromatasehemmer keine Ergebnisse vor und die bekannten Daten zur chemoendokrinen Therapie mit Tamoxifen dürfen nicht auf Aromatasehemmer übertragen werden.

Auch wird heute fast ausnahmslos bei der Indikation zu einer Chemotherapie ein taxanhaltiges Regime eingesetzt. Vor dem Hintergrund der nachgewiesenen negativen
Beeinflussung der Zellproliferation durch die Aromatasehemmer und vor dem Hintergrund der besseren Wirksamkeit der Chemotherapie bei hochproliferierenden Tumoren (luminal A versus luminal B!) spricht trotz der offenen Fragen vieles für den Vorteil einer sequenziellen vor einer simultanen chemoendokrinen Therapie.

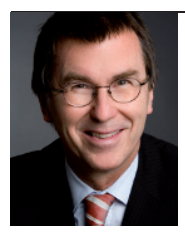

Prof. Dr. med. Volker Möbus Klinik für Gynäkologie und Geburtshilfe, Klinikum Frankfurt Höchst volker.moebus@klinikumfrankfurt.de

\title{
Neoadjuvante Therapie: Vorteile für Trastuzumab
}

\section{Verglichen mit Lapatinib führt Trastuzumab, kombiniert mit Chemotherapie, in der neoadjuvanten Behandlung von Brustkrebs zu einer höheren Rate pathologischer Komplettremissionen.}

egenüber standen sich in der PhaseIII-Studie der German Breast Group und der Arbeitsgemeinschaft Gynäkologische Onkologie-Breast zwei neoadju- vante Regimes für das HER2-positive Mammakarzinom: vier Zyklen Epirubicin plus Cyclophosphamid (EC) und vier Zyklen Docetaxel, je ergänzt um entweder

\begin{tabular}{|c|c|c|c|c|}
\hline \multicolumn{3}{|l|}{ Subgruppe } & $\begin{array}{c}\text { Odds Ratio } \\
\text { (95\%-Konfidenzintervall) }\end{array}$ & $\begin{array}{c}\text { Test zur } \\
\text { Interaktion }\end{array}$ \\
\hline \multirow{2}{*}{\multicolumn{2}{|c|}{$\begin{array}{l}\text { Alter (Jahre) } \\
<<0\end{array}$}} & & & 0,41 \\
\hline & & 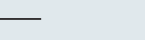 & $0,46(0,17-1,26)$ & \\
\hline$\geq 40$ & & - & $0,73(0,49-1,07)$ & \\
\hline \multicolumn{2}{|l|}{ T-Stadium } & & & 0,87 \\
\hline \multicolumn{2}{|l|}{ CT1-3 } & & $0,67(0,45-0,997)$ & \\
\hline \multicolumn{2}{|l|}{ cT4 } & & $0,72(0,32-1,65)$ & \\
\hline \multicolumn{2}{|l|}{$\mathrm{N}$-Stadium } & & & 0,3 \\
\hline \multicolumn{2}{|l|}{ cNO } & - & $0,60(0,32-1,13)$ & \\
\hline \multicolumn{2}{|l|}{$\mathrm{cN}+$} & - & $0,72(0,46-1,13)$ & \\
\hline \multicolumn{2}{|l|}{ Stadium } & & & 0,42 \\
\hline \multicolumn{2}{|c|}{ operabel } & & $\begin{array}{l}0,63(0,42-0,94) \\
0,92(0,40-2,15)\end{array}$ & \\
\hline \multicolumn{2}{|c|}{ Histologischer Typ } & & $0,92(0,40-2,13)$ & 0,18 \\
\hline \multicolumn{2}{|l|}{$\begin{array}{l}\text { duktal oder andere } \\
\text { lobulär }\end{array}$} & & $\leftrightarrow \begin{array}{c}0,65(0,45-0,94) \\
2,67(0,33-21,3)\end{array}$ & \\
\hline \multicolumn{2}{|l|}{ Grad } & & & 0,45 \\
\hline \multicolumn{2}{|l|}{1 oder 2} & & $0,78(0,46-1,32)$ & \\
\hline \multicolumn{2}{|l|}{3} & & $0,59(0,36-0,97)$ & \\
\hline $\begin{array}{l}\text { Hazard Ratio } \\
\text { negativ } \\
\text { positiv }\end{array}$ & & & $\begin{array}{l}0,82(0,50-1,36) \\
0,53(0,31-0,91)\end{array}$ & 0,40 \\
\hline \multirow[t]{3}{*}{ Gesamt } & & & $0,68(0,47-0,97)$ & \\
\hline & $\begin{array}{llll} & 1 & 1 \\
0,4 & 0,6 & 0,8 & 1,0\end{array}$ & 2,0 & & \\
\hline & pCR höher unter Trastuzumab & $\mathrm{pCR}$ höher unt & Lapatinib & \\
\hline
\end{tabular}

Abb.: Trastuzumab zusätzlich zur Chemotherapie führte zu einer höheren Rate vollständigen pathologischen Ansprechens als die Zugabe von Lapatinib.
1. Trastuzumab $6 \mathrm{mg} / \mathrm{kg}$ i.v. (Startdosis von $8 \mathrm{mg} / \mathrm{kg}$ an Tag 1 des ersten ECZyklus, ECH-TH-Gruppe) oder

2. Lapatinib $1.000-1.250 \mathrm{mg} / \mathrm{Tag}$ oral (ECL-TL-Gruppe).

Bei den 620 Patientinnen an 127 Zentren waren HER2-positive Tumoren diagnostiziert worden, die lokal fortgeschritten (T3/4a-d), Hormonrezeptor-positiv im Stadium T2N+ bzw. T1pNSLN (mit pathologisch positivem Sentinel-Lymphknoten) oder Rezeptor-negativ gewesen waren. Primärer Endpunkt war das vollständige pathologische Ansprechen auf die neoadjuvante Therapie (Abb.). Das erreichten 30,3\% der Patientinnen in der ECH-TH-und $22,7 \%$ in der ECL-TLGruppe, was einer Odds Ratio von 0,68 zuungunsten von Lapatinib entspricht ( $\mathrm{p}$ $=0,04)$. Unter Trastuzumab waren mehr Ödeme und Dyspnoen zu beobachten, unter Lapatinib mehr Diarrhöen und Hautausschläge.

Fazit: Vollständige pathologische Remissionen waren nach einer Chemotherapie mit Lapatinib signifikant seltener als nach Trastuzumab. Die Forscher raten daher, Lapatinib außerhalb klinischer Studien nicht als alleinige Anti-HER2-Behandlung in Kombination mit neoadjuvanter Chemotherapie zu geben. Robert Bublak

Untch $\mathrm{M}$ et al. Lapatinib versus trastuzumab in combination with neoadjuvant anthracycline-taxane-based chemotherapy (GeparQuinto, GBG 44): a randomised phase 3 trial. Lancet Oncol. 2012;13(2):135-44. 